\title{
Organisation sociale et zootechnique de la gestion des produits laitiers en milieu sahélien : la sphère laitière. Cas du delta du fleuve Sénégal *
}

\author{
C. Corniaux ${ }^{1}$
}

\begin{abstract}
Mots-clés
Lait - Produits laitiers - Traite Organisation - Famille - Modèle Sénégal.
\end{abstract}

\begin{abstract}
Résumé
En milieu sahélien, la concession est une organisation sociale commune mais complexe. L'illustration en est donnée pour la gestion des produits laitiers en milieu pastoral et agropastoral. Sur la base d'un travail de terrain mené auprès d'éleveurs du delta du fleuve Sénégal, une schématisation de l'organisation sociale et zootechnique de cette gestion a été établie. La traite est le moment crucial de la gestion du lait. Elle permet de déterminer les contours de la sphère laitière qui regroupe à la fois les animaux en production et les individus qui les gèrent. Le modèle proposé permet de distinguer différents niveaux décisionnels : celui du berger-trayeur qui décide des quantités traites (production) et celui des collectrices qui décident individuellement du devenir du lait trait (autoconsommation, don, commercialisation). En outre, le présent modèle permet d'éviter le piège qui consiste à assimiler le gestionnaire du troupeau ou le chef de concession à un pilote d'une exploitation laitière.
\end{abstract}

\section{INTRODUCTION}

La concession, base de l'organisation sociale des producteurs sahéliens, est généralement perçue comme une exploitation familiale. En dépit de la mise en garde déjà ancienne de certains auteurs (6), le chef de famille a souvent été considéré comme le chef d'exploitation. Ainsi en matière d'élevage, le gestionnaire des troupeaux de la concession, généralement l'aîné de la famille, est systématiquement assimilé à un "pilote », pour reprendre le vocabulaire couramment utilisé dans les recherches d'inspiration systémique (8). En tant que tel, il représente un interlocuteur privilégié pour les techniciens en charge de la mise en œuvre des politiques d'élevage. C'est aujourd'hui le cas dans la promotion d'innovations techniques visant le développement de la production laitière. Pourtant, l'efficience toute relative de ces politiques depuis des décennies amène à se réinterroger sur les niveaux décisionnels en matière de gestion des produits laitiers et sur l'occurrence du choix du gestionnaire de troupeau en tant qu'interlocuteur privilégié au sein de la concession.

Aussi, sur la base d'un travail de terrain mené auprès d'éleveurs du delta du fleuve Sénégal (3), l'auteur propose d'établir un schéma

1. Cirad, UPR Systèmes d'élevage, BP 1813, Bamako, Mali.

Tél. : +2232214293 ; e-mail : corniaux@cirad.fr organisationnel de cette gestion des produits laitiers au sein de la concession. Après sa présentation, seront évalués ses atouts et ses limites avant d'apprécier son intérêt dans l'identification du « producteur laitier» en milieu sahélien.

\section{MATERIEL ET METHODES}

\section{Zone d'étude}

L'étude a été menée dans le delta du fleuve Sénégal, entité géographique, comprise entre $15,8-16,5^{\circ} \mathrm{N}$ et $15,7-16,5^{\circ} \mathrm{O}$. Il est délimité au nord par le fleuve, à l'ouest par l'océan Atlantique, à l'est par le lac de Guiers et au sud par une ligne Mpal - Keur Momar Sarr. Sa superficie est de l'ordre de $5000 \mathrm{~km}^{2}$ (figure 1).

Le découpage morphopédologique peut être présenté sous deux grands ensembles. Le premier est constitué par la partie dunaire ou diéri, largement majoritaire au sud de l'axe routier. L'élevage

* Le présent article est issu d'un travail de thèse soutenue en juin 2005 : Corniaux C., 2005, Gestion technique et gestion sociale de la production laitière : les champs du possible pour une commercialisation durable du lait - Cas des systèmes d'élevage actuels du delta du fleuve Sénégal. Thèse Doct., INA-PG, Ecole doctorale Abies, Paris, France, 250 p., www.epe.cirad.fr 


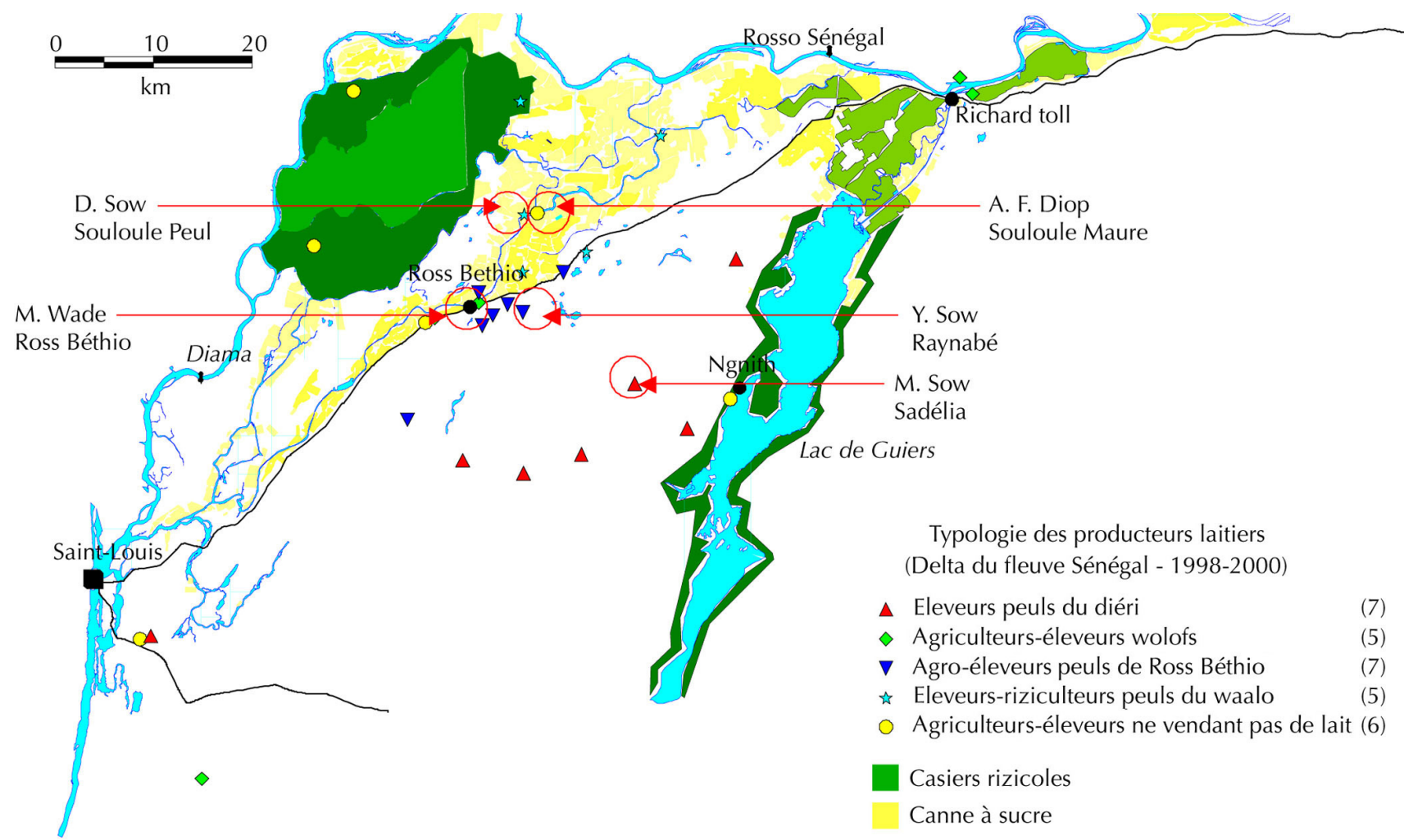

Figure 1 : localisation des cinq concessions de l'étude dans le delta du fleuve Sénégal.

et l'agriculture pluviale y sont pratiqués. En bordure du lac et du fleuve, sont présentes des cuvettes de décantation appelées hollaldé, vastes zones dépressionnaires plates et argileuses. Elles forment le waalo. Des aménagements hydroagricoles y ont été massivement implantés depuis les années 1960.

Le climat, de type sahélien, comprend trois principales saisons : l'hivernage ou saison des pluies (de juillet à septembre), la saison sèche froide (octobre à mars) et la saison sèche chaude, ou période de soudure, difficile sur le plan alimentaire pour les troupeaux (avril à juin-juillet). La zone se situe autour de l'isohyète $250 \mathrm{~mm}$. La principale caractéristique de la pluviométrie est la très grande irrégularité interannuelle des précipitations. La température moyenne annuelle est de $25{ }^{\circ} \mathrm{C}$ avec une variabilité importante. La végétation, de type sahélien, est influencée par le fleuve Sénégal et les remontées salines.

\section{Méthode}

\section{Schéma de base}

L'organisation des sociétés sahéliennes est décrite dans la littérature. Elle est le fait d'anthropologues, de sociologues et de géographes. C'est le cas des sociétés lignagères (10) présentes dans le delta du fleuve Sénégal et plus particulièrement des Peuls. Ainsi, les auteurs $(1,5,12)$ s'accordent à structurer leur organisation sociale en trois principaux niveaux (figure 2) :

- la concession (galle chez les Peuls) ; c'est l'unité familiale. Que ce soit chez les Peuls, les Wolofs ou les Maures, l'organisation visible est celle de la concession, correspondant à un segment de lignage et rassemblant sous la tutelle d'un père ou d'un aîné plusieurs hommes adultes, leurs épouses et leur progéniture. Mais il ne faudrait pas d'emblée, comme c'est trop souvent le cas dans les documents techniques, assimiler cette concession à une exploitation agricole. Il s'agit plutôt d'une unité de résidence, d'un cadre de solidarité et d'entraide :
- le foyer (fooyere chez les Peuls) ; c'est l'unité de production. Elle comprend le mari, sa femme (plusieurs en cas de polygamie) et ses enfants. Il s'agit du cadre domestique d'activités et de coopération dans le travail. C'est à ce niveau que la majorité des décisions sont prises ; - la case (suudu chez les Peuls); chaque épouse dispose de sa case. Elle y habite avec ses enfants et y reçoit son mari.

Le regroupement de plusieurs concessions forme un campement (wuro chez les Peuls), un hameau ou un village.

La correspondance de ces niveaux de gestion sociale avec celle des animaux est remarquable. On notera notamment que chez les Peuls, la cohésion est soulignée par le terme dudal qui ne fait pas de distinction entre les deux à l'échelle de la concession (1).

Ce schéma organisationnel a servi de base de travail pour déterminer les niveaux hiérarchiques dans la concession. Il a été

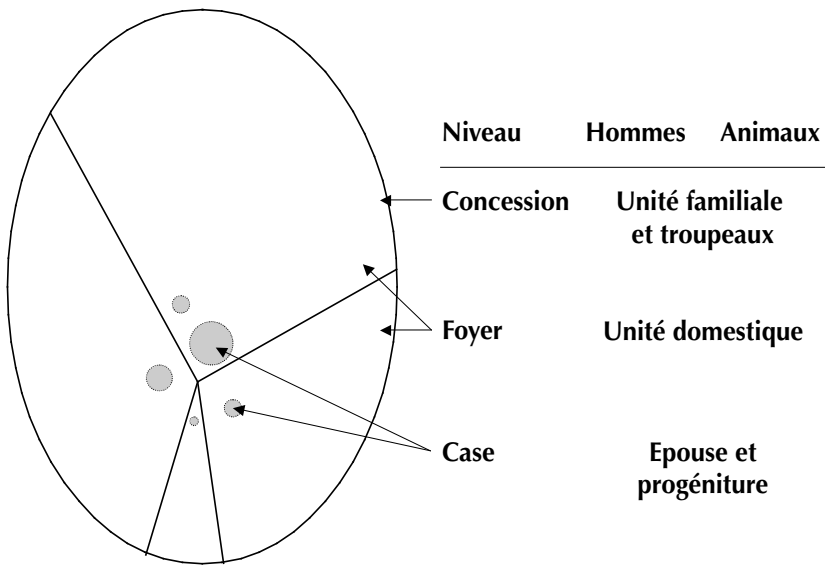

Figure 2 : schéma de l'organisation sociale et zootechnique des concessions. 
amendé en fonction des niveaux décisionnels mis en lumière en matière de gestion des produits laitiers.

\section{Monographies}

A l'échelle de la concession, la question directrice a été la suivante : qui décide quoi en matière de gestion des produits laitiers ? La multiplicité des niveaux décisionnels, la difficulté à tracer les contours des troupeaux ou encore la plasticité de la structure des concessions ont conduit à faire des choix méthodologiques. Les monographies de situations singulières ont été privilégiées. Elles ont permis d'établir un cadre d'analyse original susceptible de rendre compte de situations diverses. En outre, une monographie établie sur la base d'un suivi systématique et prolongé dans le temps a permis d'accéder à un niveau d'informations impossible à atteindre dans le cadre d'enquêtes touchant plusieurs dizaines d'éleveurs.

Dès le départ, il est apparu opportun de concentrer les efforts sur la traite. Elle représentait un nœud d'articulation entre une population animale et une population humaine qu'il fallait définir afin de déterminer les limites du système d'étude, appelé plus loin la « sphère laitière ». Dans ce cadre, l'élément clé a été le bergertrayeur, fonction essentiellement tenue par des hommes dans le delta du fleuve Sénégal. C'est lui qui chaque jour posait les jalons des deux populations. En observant le berger au moment de la traite, il était possible de noter à la fois les animaux allaitants et la destination du lait trait, chaque récipient de collecte correspondant à une personne. Chaque destinataire pouvait ensuite être interrogé sur le devenir du lait trait.

Le caractère évolutif et cyclique de la production laitière a permis de faire un suivi mensuel sur une année entière de l'hivernage 2002 à l'hivernage 2003. Ce suivi était ciblé sur les flux de produits animaux, notamment le lait, entre les populations humaine et animale des concessions. La mesure de ces flux, sur le plan quantitatif et qualitatif (nature : autoconsommation, don, vente ; statut des hommes et des animaux), a permis d'apprécier le poids décisionnel de chaque individu au sein du système d'étude.

Concrètement, cinq situations ont été prises en compte (figure 1). Elles correspondaient à des groupes de producteurs laitiers (3) :

- éleveurs peuls du diéri, Moutar Sow ;

- agroéleveurs peuls de Ross-Béthio, Yérodi Sow ;

- éleveurs-riziculteurs peuls du waalo, Diary Sow ;

- éleveurs maures ne vendant pas de lait, Abdou Fata Diop ;

- éleveurs wolofs, Amadou Wade.

Les noms correspondent à ceux des chefs de concession.

\section{RESULTATS}

\section{Schéma général}

Au sein de la concession, sont apparus rapidement deux niveaux décisionnels majeurs dans la gestion du lait. Le premier était celui du berger-trayeur. C'est lui qui déterminait les animaux à traire et le temps de la traite. Chez les Peuls, il s'agissait généralement du fils du chef de la concession. Sa fonction de berger le poussait à préserver l'état des vaches en lactation ainsi que celui des veaux. La règle était que le veau était prioritaire sur la traite. En saison sèche, dans des conditions d'alimentation déficitaires, le bergertrayeur était par conséquent enclin à interrompre la traite.

Le second était celui des destinataires du lait trait. Ainsi dans les concessions peules, la quasi-totalité des femmes, les collectrices, recevaient une part de lait. Elles décidaient de son devenir en choisissant entre l'autoconsommation, le don et la vente. Leur intérêt résidait par conséquent dans la traite la plus complète possible des animaux laitiers.

Ces deux niveaux décisionnels peuvent être intégrés au schéma de base (figure 2) pour donner un schéma général de l'organisation sociale et zootechnique de la gestion du lait à l'échelle de la concession (figure 3). Ainsi, le niveau de la collectrice épouse parfaitement celui de la case. En revanche, le niveau du berger trayeur est inédit par rapport au schéma organisationnel initial de la concession. Il rassemble toutes les cases de femmes mariées, indépendamment des limites des foyers. Il détermine la sphère laitière. Elle regroupe à la fois une population humaine, le berger-trayeur et les destinataires du lait trait, et une population animale, les femelles en lactation.

Dans cette représentation, le gestionnaire du troupeau et le chef de concession sont absents de la sphère laitière. Ils appartiennent à une sphère décisionnelle étrangère à la vente du lait. Ce constat n'est pas neutre quand on sait qu'ils représentent les interlocuteurs privilégiés des techniciens en charge du développement de la production et de la collecte de lait en milieu sahélien (cf. infra).

\section{Application du modèle aux concessions du delta du fleuve Sénégal}

Le schéma général n'est pas figé. Il s'applique et s'adapte aux situations diverses rencontrées dans le delta du fleuve Sénégal (figure 4).

\section{Prise en compte du nombre de foyers dans les concessions}

En fonction des contextes, les niveaux décisionnels pouvaient s'emboîter, comme chez A. Wade (Wolof) et chez A.F. Diop

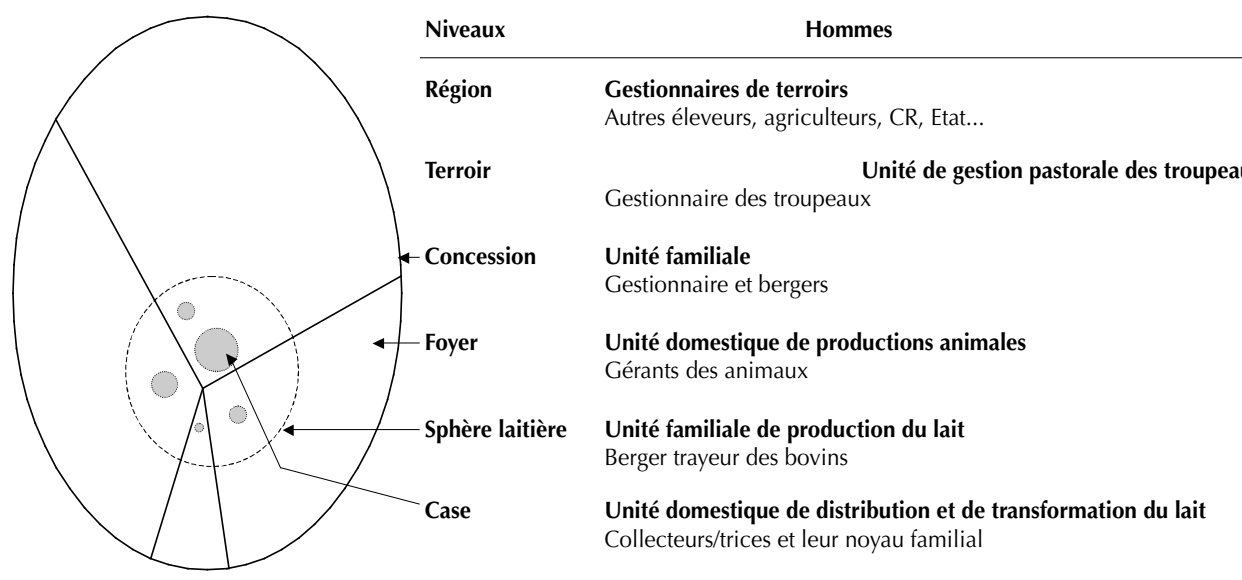

Addition de troupeaux

Unité zootechnique de production Troupeaux gérés par l'unité familiale

Unité zootechnique de production du lait Femelles allaitantes et suitées

Noyau élémentaire de femelles laitières traites

Figure 3 : schéma de l'organisation sociale et zootechnique de la gestion du lait à l'échelle des concessions. 
(Maure) qui cumulaient plusieurs fonctions puisqu'ils étaient à la fois gestionnaires de troupeaux et collecteurs de lait. A.F. Diop était même périodiquement trayeur. Dans leur cas c'était l'unité domestique qui venait se superposer à l'unité familiale. Ils maîtrisaient dès lors la totalité du processus de production et de commercialisation du lait, à l'image d'un véritable producteur laitier pilote de son exploitation. Dans cette configuration, le berger-trayeur n'était qu'un salarié sous la direction de ce pilote.

Chez les Peuls, la situation était plus complexe du fait de la présence de plusieurs foyers. En fait, plus le nombre d'unités domestiques augmentait dans la concession, plus les niveaux d'organisation étaient séparés. C'était le cas chez Moutar Sow et chez Yérodi Sow, respectivement à Sadiélia et à Raynabé, dans la partie pastorale du delta du fleuve Sénégal. Ces gestionnaires de troupeaux avaient un pouvoir limité et indirect sur la sphère laitière. Il dépendait notamment de leur décision de départ en transhumance avec tout ou partie des vaches laitières, cette décision étant conditionnée par la pluviométrie annuelle. Dans le delta, du fait de la sédentarisation des hommes et de la présence de parcours postculturaux, la transhumance est néanmoins réduite dans l'espace et dans le temps (2). Dans ce type d'organisation sociale, le réel pouvoir sur le lait revient finalement au berger-trayeur qui décide de traire, c'est-à-dire de produire, et aux collectrices qui décident du devenir du lait trait et en particulier de sa vente.

Chez Diary Sow à Souloule Peul, au cœur des casiers rizicoles, la situation était intermédiaire. Il n'y avait que trois foyers dans la concession de cette veuve : le sien, celui de sa mère et celui de son fils (berger-trayeur). La gestion du lait faisait donc intervenir moins d'individus, de surcroît directement affiliés. Diary, chef de sa concession, confiait néanmoins la conduite aux pâturages de ses animaux à Seydi Ka, le gestionnaire des troupeaux du wuro. Par conséquent, si son autonomie sur les produits de la traite était grande, elle ne maîtrisait toujours pas les départs éventuels en transhumance.

\section{Gestion individuelle du lait collecté}

Le caractère collectif de la traite est clairement souligné par la schématisation proposée (figure 4). Elle marque tout autant le caractère individuel de la décision relative au devenir du lait trait.

Pour les destinataires du lait trait, les cercles du niveau décisionnel présentaient un diamètre variable en fonction du nombre d'animaux traits ou des quantités de lait collecté (figure 4). Ainsi, chez les Peuls, il était possible de hiérarchiser l'importance des collectrices au sein de leur concession. Diary, Djenaba et Djuma apparaissaient comme les mieux dotées respectivement à Souloule, Raynabé et Sadiélia. En particulier, Djuma, première femme d'Abdul (berger-trayeur de Sadiélia), recevait sensiblement plus de lait que sa coépouse Fatimata. Certaines collectrices apparaissaient marginalisées, comme Fatimata à Souloule, Djenaba2, Mary, Aby, Aminata et Awa à Raynabé, Diary, Aminata et Awa à Sadiélia. En revanche, en l'état il n'était pas possible de distinguer leur marge de manœuvre dans la mesure où le statut des animaux de la sphère laitière n'apparaît pas dans la schématisation (cf. infra).

Au-delà d'une hiérarchisation au sein des concessions, la figure 4 permet également de comparer les situations entre elles. Le biais de l'évaluation de la production laitière à l'échelle de l'exploitation

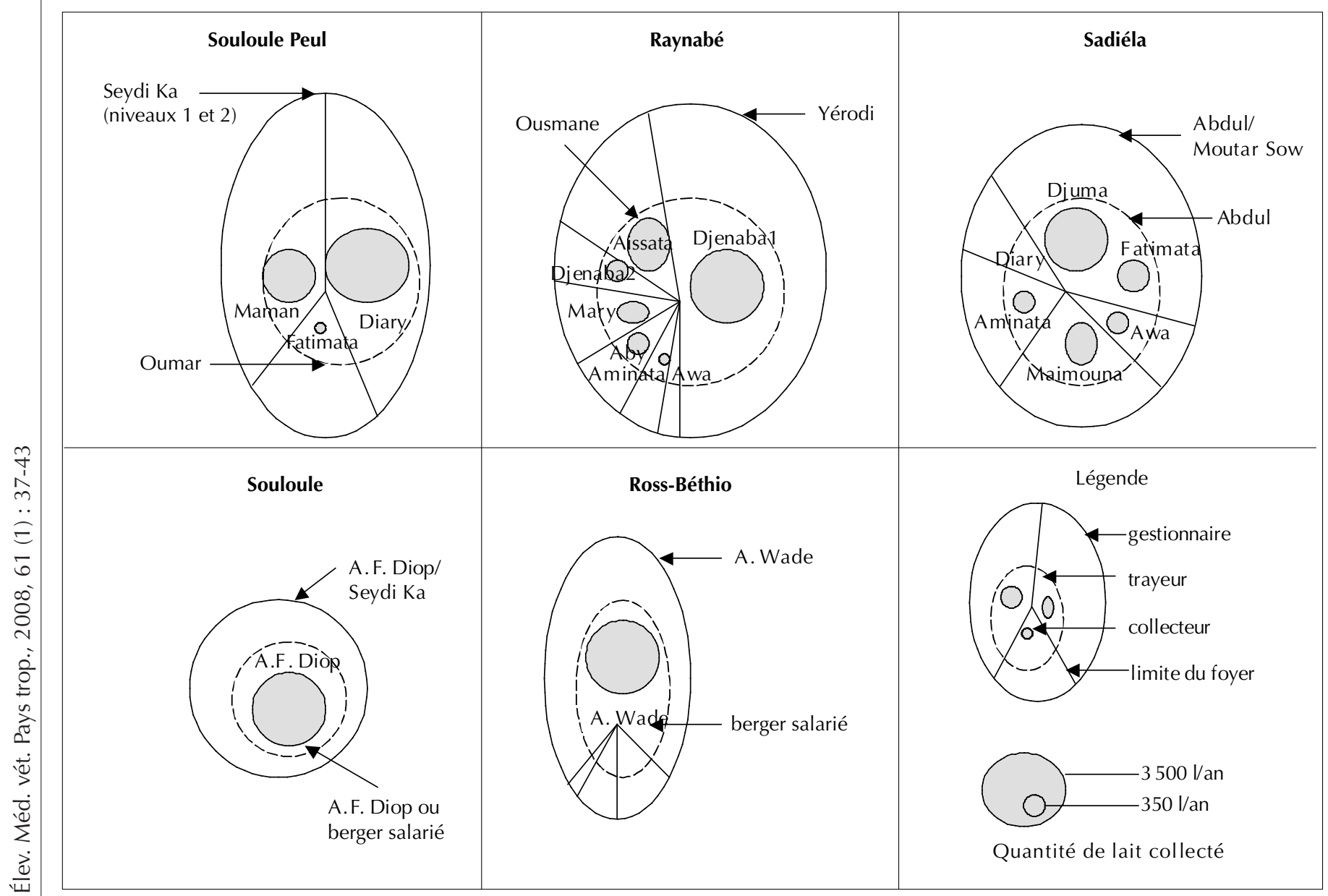

Figure 4 : représentation de la gestion du lait de vache dans cinq concessions du delta du fleuve Sénégal (d'après Corniaux, 2005). 
a toutefois été sciemment évité. Ainsi dans le cas des concessions wolof ou maure qui produisaient sensiblement moins que les concessions peules, il est apparu que la collecte d'A.F. Diop, le Maure, et celle d'A. Wade, le Wolof, étaient analogues à celles des meilleures collectrices peules, Diary, Djenaba et Djuma. C'est donc à cette échelle qu'il était pertinent de comparer les stratégies de chacun d'entre eux en matière de destination des produits laitiers.

\section{DISCUSSION}

\section{Intérêts et limites de la schématisation}

L'enjeu scientifique de la schématisation proposée était de construire un modèle original d'analyse et de représentation qui permettait d'apprécier les règles de gestion du lait à l'échelle de la concession. Le modèle proposé a permis d'identifier les foyers comme les unités décisionnelles de la gestion du lait. Ils étaient néanmoins insérés dans une structure familiale, la concession, qui gérait les parcours et les déplacements des troupeaux. Cette dernière pouvait dès lors interférer sur la politique laitière des ménages. Dans son choix des vaches à traire, le berger-trayeur représentait le modérateur entre les deux entités. La décision de la commercialisation du lait était quant à elle prise à l'intérieur du foyer. Elle résultait d'un compromis discuté entre un époux souvent propriétaire des animaux et sa ou ses épouses, généralement détentrices du droit sur le lait (4).

L'intérêt de ce type de modèle est d'être adaptable aux différentes situations rencontrées dans le delta du fleuve Sénégal. Ainsi, il permet de schématiser à la fois l'enchâssement des niveaux décisionnels de grandes concessions peules du diéri et, à l'autre extrémité, la concentration de ces niveaux décisionnels autour d'un seul individu dans le cas du troupeau confié chez un Wolof du waalo. Cette souplesse indique que ce modèle peut vraisemblablement être mobilisé dans diverses situations agropastorales.

Ce modèle permet aussi d'appréhender les modifications de la structure de la gestion du lait. Il intègre notamment les cas de scission des concessions, si fréquents en milieu agropastoral, qui s'opère à la suite du départ d'un ou de plusieurs foyers de la concession mère $(1,5,10)$. Il permet par conséquent d'apprécier les évolutions et les changements structurels de cette gestion. En fonction de la représentation de la taille de ses éléments, il peut même donner une idée précise des modifications quantitatives qui les accompagnent, en termes de nombre d'animaux et de flux laitiers. Pour des suivis à plus long terme, ce modèle pourrait devenir un outil intéressant pour souligner les trajectoires d'évolution des exploitations familiales productrices de lait.

En revanche, il ne permet pas de distinguer l'importance relative des différents troupeaux laitiers au sein de la concession, à moins de faire deux schémas distincts, l'un pour les bovins, l'autre pour les caprins. Surtout, la schématisation actuelle ne fait pas apparaitre le statut des animaux, si important dans la détermination des règles de la gestion du lait. A titre d'exemple, Corniaux et coll. (4) notent que Diary Sow, à Souloule Peul, est veuve et qu'elle possède les animaux hérités de son mari en plus des animaux de sa dot. A l'inverse, Djuma de Sadiélia possède très peu d'animaux et dépend de l'allocation des vaches de son époux, de surcroît polygame. Diary dispose donc d'un degré d'autonomie plus conséquent que celui de Djuma dans la gestion de ses produits laitiers. Pour préciser le modèle, on pourrait imaginer de fractionner les cercles des collecteurs en fonction de ce statut. Mais la représentation risque d'être partielle et peu lisible. Il s'agit probablement d'une limite majeure dans son efficacité. Une autre limite est celle de l'absence de formalisation du niveau de la consommation du lait. Mais la variabilité de la mise en commun des plats à base de lait rend particulièrement difficile sa représentation dans le présent modèle.
Par ailleurs, au-delà de son utilité pratique, ce modèle permet de revisiter les notions de «pilote » et d' « exploitation » largement utilisées dans les études systémiques (8) mais inadaptées au milieu sahélien (et davantage encore quand on s'intéresse à la production laitière) (7). Il évite le piège d'identifier le gestionnaire du troupeau comme étant le pilote principal de la gestion du lait dans le cadre de son exploitation agricole. L'exploitation agricole, au sens occidental du terme, n'existe pas en milieu sahélien. Une différence fondamentale avec le modèle occidental de l'exploitation réside dans son activité. Au Sahel, les structures ne sont pas exclusivement tournées vers l'exploitation marchande des produits. L'autoconsommation et le don y ont une place conséquente. Quant au gestionnaire, il n'intervient souvent que de façon partielle et indirecte (alimentation et déplacement des troupeaux laitiers). Même lorsque les animaux lui appartiennent, le choix des vaches à traire, des quantités traites et des quantités vendues ne relèvent pas de son unique autorité. Ce sont les trayeurs et les collecteurs qui sont au cœur du pouvoir décisionnel concernant les flux de lait.

En outre, la schématisation est facile et rapide à établir. Si un suivi régulier et relativement lourd sur un minimum d'une année est nécessaire pour son édification, son application ne demande qu'un ou deux passages au moment de la traite dans les concessions ciblées. Il est conseillé de prévoir des visites en hivernage quand un maximum d'individus et d'animaux laitiers sont concernés. La taille des cercles correspond alors à la production mesurée le jour du passage.

Finalement, ce type de représentation permet d'apprécier instantanément la complexité des rapports décisionnels pour la gestion du lait au sein d'une concession. En marquant clairement le nombre de foyers et les cas de polygamie, il est possible de se faire une idée précise sur les rapports de pouvoir entre les individus. De surcroît, un niveau de sophistication supérieur est atteint dès lors que les différents cadrans et cercles reflètent la taille des troupeaux et des flux laitiers.

\section{Une organisation évolutive}

La plasticité du modèle proposé dépend de deux principaux facteurs. Le premier a déjà été décrit et correspond au changement du nombre de foyers présents dans chaque concession (figures 4 et 5). Le second est relatif au nombre de trayeurs impliqués (figure 5). Il arrive en effet que les destinataires du lait, les collectrices chez les Peuls, se chargent de la traite de leurs propres animaux. Le cas est bien décrit en milieu pastoral $(5,8,12)$. En milieu agropastoral, la situation est moins commune. Pour les bovins, elle se rencontre néanmoins en hivernage quand le nombre de vaches en production dépasse les capacités du trayeur. En revanche, la traite des chèvres demeure une prérogative des femmes dans les concessions (4). Par conséquent, les deux organisations peuvent coexister dans une même concession en fonction du troupeau concerné ou de la période de l'année.

En fait, cette coexistence marque sans doute la transition organisationnelle dans laquelle se trouvent les concessions du milieu agropastoral. L'agriculture implique une sédentarisation des personnes et une diversification des activités et des revenus. En milieu irrigué, la précarité alimentaire est sensiblement réduite à la fois pour les hommes et leurs troupeaux. Ces éléments, parmi d'autres (exode rural, limitation de la pratique de la polygamie...), jouent en faveur d'un fractionnement des concessions et d'une diminution de leur taille, c'est-à-dire d'une réduction du nombre de foyers $(10,12,13)$. Elle va de pair avec la limitation de la main d'œuvre et, par conséquent, avec une redistribution des tâches au sein de la concession (11). Les agriculteurs résolvent la question de la garde des troupeaux en les confiant à des bergers salariés, qui se chargent alors de la traite. Ainsi quand on passe du milieu pastoral au milieu agropastoral, la traite initialement effectuée par des femmes revient au berger- 


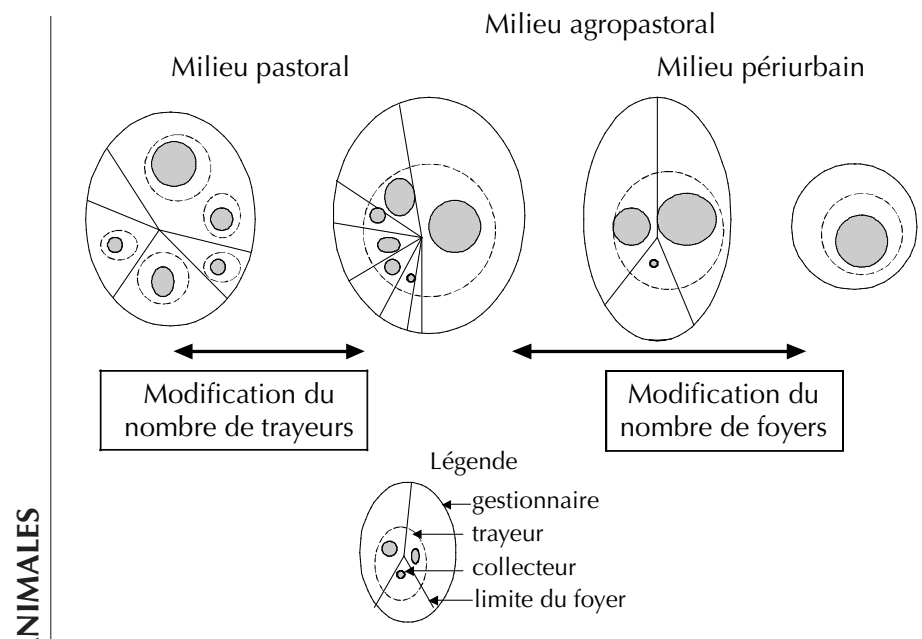

Figure 5 : schématisation de l'évolution de l'organisation sociale de la gestion du lait en milieu subsaharien à l'échelle des concessions.

trayeur. Mais le véritable enjeu du changement de la règle ne relève probablement pas d'un problème de main d'œuvre. Dupire estime que la division sexuelle du travail chez les Peuls répond à une situation économique déterminée (5). Or, la sédentarisation s'accompagne généralement d'une ouverture du marché laitier avec la proximité de communautés non pastorales, avec l'urbanisation, avec la présence de structure de collecte et avec l'amélioration des voies de communication. Elle est également synonyme de réduction de la mobilité des troupeaux et d'un accès facilité aux services (soins vétérinaires, aliments du bétail). La combinaison de ces facteurs favorise une augmentation des quantités traites. Les hommes en charge du troupeau, les gestionnaires et les bergers ne sont pas prêts de remettre en cause leur statut social qui leur impose de protéger les veaux. Le meilleur moyen est de contrôler la traite en l'effectuant eux-mêmes. En outre, en milieu pastoral nomade ou transhumant, les collectrices assurent la traite parce qu'elle correspond à une tâche domestique destinée à subvenir aux besoins d'autoconsommation. Même quand il y a vente, le bénéfice est immédiatement réinvesti pour couvrir les dépenses du foyer. Quand il y a sédentarisation et augmentation de la part du lait vendu, tout change. L'autonomie financière des femmes peut s'élargir. On touche à l'équilibre des pouvoirs dans la concession. Autrement dit, on touche à l'autorité masculine. Dans un système que les hommes dirigent, il est dès lors moins surprenant de les voir enclins à modifier la règle en s'octroyant le niveau décisionnel de la traite.

Pour autant, la prise en charge exclusive de la traite par un berger ou la diminution du nombre de foyers ne remettent pas fondamentalement en cause l'organisation de la concession. Pouillon (9) confirme que les scissions chez les pasteurs peuls ne sont en rien synonymes de déflagration sociale. L'objectif n'est pas de casser le système mais de le prendre en mains. Il s'agit aussi d'adopter une organisation sociale éprouvée, capable de se perpétuer dans un environnement hostile. Les risques liés aux aléas climatiques et à la perte de solidarité entre les concessions limitent en fait les possibilités de changements plus radicaux du système.

Pour la production laitière, le véritable changement réside dans la mise en place d'unités de production spécialisées, pilotées par un chef d'exploitation. Cette situation existe en milieu périurbain (figure 5) mais elle demeure marginale dans le delta du fleuve Sénégal et plus largement en milieu sahélien. Le modèle de la concession regroupant plusieurs foyers reste l'organisation de base. Il évolue dès lors de façon réversible au gré des phases de sédentarisation, de l'essor ou des échecs des opérations de collecte laitière, et des cycles de pastoralisation et de dépastoralisation.

\section{Adéquation avec les politiques laitières et innovation chez les producteurs laitiers}

La figure 4 montre la complexité organisationnelle de la gestion du lait trait, notamment en milieu pastoral. La vente garde toutefois un caractère individuel. Autrement dit, prétendre décrire la stratégie de vente de lait d'un producteur sur la base de la production globale à l'échelle de sa concession est une erreur fondamentale, surtout en milieu pastoral. La vente, l'autoconsommation et le don sont réalisés sur la base d'une collecte individualisée. Les revenus du lait sont individualisés. Les charges le sont tout autant. En particulier, les dépenses relatives à la complémentation et aux soins vétérinaires destinés aux animaux en lactation reviennent aux bénéficiaires de la traite, c'est-à-dire aux bénéficiaires de l'argent $\mathrm{du}$ lait. Les gestionnaires du troupeau ou les chefs de concessions qui agissent de façon collective sur le cheptel ne sont pas dans cette sphère décisionnelle. Ils ne gèrent ni les recettes ni les dépenses relatives au lait : ce ne sont pas des producteurs laitiers. Or ils sont bien souvent les interlocuteurs privilégiés des politiques visant le développement de la production laitière.

Dès lors, il ne faut pas s'étonner de la faible réceptivité du milieu pastoral sahélien aux innovations techniques proposées par ces politiques. Il n'est pas tant question d'immobilisme (9) que d'inadéquation de ces politiques avec les stratégies des interlocuteurs. Il est avant tout question de lever des contraintes techniques ciblées sur l'amont de la filière. Elles recouvrent quatre aspects : l'alimentation, la génétique, la reproduction et l'hygiène. Parmi eux, l'alimentation est même considérée, à juste titre, comme le principal facteur limitant. Mais cette approche techniciste a montré ses limites depuis des décennies, malgré des référentiels maîtrisés par la recherche et les services de diffusion technique. N'y a-t-il pas lieu de reconnaître humblement un décalage avec les attentes et les possibilités des pasteurs ? L'expérience des inséminations artificielles illustre remarquablement cette discordance. " Il vaut mieux avoir deux vaches à $15 \mathrm{l} / \mathrm{j}$ que 30 à $1 \mathrm{l} / \mathrm{j}$ » peut-on entendre régulièrement de la bouche de techniciens. Pourtant, comment pourrait-on exiger d'un gestionnaire de troupeaux, l'interlocuteur privilégié, de supprimer des animaux alors même qu'ils appartiennent à différentes personnes, que leur grand nombre est synonyme de sécurité économique et de sécurité face aux aléas climatiques et, qu'enfin, de ce nombre dépend le prestige et le rôle des propriétaires notamment dans les cérémonies traditionnelles ? Il est invraisemblable de demander à ce gestionnaire d'abattre ou de se séparer des animaux confiés alors que sa fonction lui dicte précisément de les préserver ou, mieux, de faire fructifier le capital.

Il en est de même dans les discours adressés aux gestionnaires pour une complémentation alimentaire ciblée ou pour les soins vétérinaires à apporter aux animaux laitiers. Ces pratiques sont du ressort des individus qui gèrent le lait. Les gestionnaires n'agissent qu'au niveau du collectif, notamment dans l'achat groupé et la distribution d'aliments de sauvegarde en fin de saison sèche.

$\mathrm{Au}$ fond, les innovations techniques ne peuvent trouver un écho favorable que dans les concessions où l'organisation sociale de la gestion du lait est la plus souple. On la rencontre en milieu agropastoral ou en milieu périurbain (figure 5). Dans ce cadre, la complémentation des vaches laitières est commune (4). Pratiquée dès le début de la saison sèche, elle vise l'augmentation de la production de lait destiné à la vente. Autrement dit, on se trouve ici devant la stratégie tant recherchée du producteur laitier, maître de l'ensemble des processus de la gestion du lait, de la production à la vente. 


\section{CONCLUSION}

L'objectif était de construire un schéma original d'analyse et de représentation qui permette d'apprécier les règles de production et de commercialisation du lait à l'échelle de la concession. Le modèle proposé a permis d'identifier les trayeurs et les collectrices comme les réels détenteurs des décisions relatives à la gestion du lait. Ils étaient néanmoins insérés dans une structure familiale, la concession, qui gérait les parcours et les déplacements des troupeaux. Cette dernière peut dès lors interférer sur la «politique laitière » des ménages. En matière de promotion des politiques laitières, la présente schématisation a permis ainsi de mettre en lumière l'inadéquation éventuelle du choix du gestionnaire de troupeau en tant qu'interlocuteur privilégié des techniciens en charge de leur application sur le terrain. A l'avenir, ils pourraient utilement user de cette schématisation, facile et rapide dans son application, en vue d'une meilleure efficience de leurs discours ou de leurs appuis techniques.

L'intérêt de ce type de modèle est enfin d'être adaptable aux différentes situations rencontrées dans le delta du fleuve Sénégal. Des études sur d'autres terrains permettront de le préciser et de le valider dans une gamme plus large de contextes sahéliens.

\section{Remerciements}

Je tiens à remercier l'équipe du centre Isra de St Louis (Sénégal) pour son appui et son accueil.

\section{BIBLIOGRAPHIE}

1. BONFIGLIOLI A.M., 1988. Dudal. Histoire de famille et histoire de troupeau chez un groupe de Wodaabe du Niger. Paris, France, Maison des sciences de l'homme, $293 \mathrm{p}$.

2. CORNIAUX C., 2001. Pratiques $d^{\prime}$ usage des ressources fourragères destinées aux troupeaux laitiers du delta du fleuve Sénégal. Liens avec la production et la commercialisation de lait. Mémoire DEA, université Paris VII, France / Cirad-emvt, Montpellier, France, 27 p.
3. CORNIAUX C., 2005. Gestion technique et gestion sociale de la production laitière : les champs du possible pour une commercialisation durable du lait. Cas des systèmes d'élevage actuels du delta du fleuve Sénégal. Thèse Doct., INA-PG, Paris, France, 250 p.

4. CORNIAUX C., VATIN F., FAYE B., 2006. Gestion du troupeau et droit sur le lait : les mécanismes de prise de décision en matière de production laitière au sein des concessions sahéliennes. Agricultures, $15: 515-522$

5. DUPIRE M., 1962. Peuls nomades. Etude descriptive des Wodaabes nigériens. Paris, France, Karthala, 440 p.

6. GASTELLU J.M., 1979. Mais où sont donc ces unités économiques que nos amis cherchent tant en Afrique? Abidjan, Côte d'ivoire, Orstom Petit-Bassam, $22 \mathrm{p}$.

7. LANDAIS E., LHOSTE P., 1990. L'association agriculture-élevage en Afrique intertropicale : un mythe techniciste confronté aux réalités de terrain. Cah. Sci. Hum., $26: 217-235$

8. LHOSTE P., MILLEVILLE P., 1986. La conduite des animaux : techniques et pratiques d'éleveurs. In : Méthodes pour la recherche sur les systèmes d'élevage en Afrique intertropicale. Maisons-Alfort, France, lemvt, p. 247-265. (Etudes et synthèses $n^{\circ} 20$ )

9. POUILLON F., 1990. Sur la «stagnation technique » chez les pasteurs nomades : les Peuls du Nord-Sénégal entre l'économie politique et I'histoire contemporaine. Cah. Sci. Hum., 26 : 173-192

10. RAYNAUT C., 1997. Sahels. Diversité et dynamiques des relations sociétés-nature. Paris, France, Grid, Karthala, 430 p.

11. SANTOIR C., 1993. Des pasteurs sur les périmètres. In : Boivin P., Dia I., Eds, Nianga, laboratoire de l'agriculture irriguée en Moyenne Vallée du Sénégal, Atelier Orstom/Isra, St Louis, Sénégal, 19-21 oct. 1993, p. 375-405.

12. TOURE O., ARPAILLANGE J., 1986. Peul du Ferlo. Paris, France, L'Harmattan, $77 \mathrm{p}$.

13. TOURRAND J.F., LANDAIS E., 1994. Aménagements hydrauliques et développement : stratégies paysannes d'adaptation dans le delta du fleuve Sénégal (1984-1991). Nat. Sci. Soc., 2 : 212-229.

Reçu le 09.08.2006, accepté le 19.03.2008

\section{Summary}

Corniaux C. Social and Zootechnical Organization of Dairy Products Management under Sahelian Conditions: The Milk Sphere. Case of the Senegal River Delta

In a Sahelian environment, the concession is a common, social, but also complex organization. An example is provided by dairy products management in pastoral and agropastoral environments. A schema of the social and zootechnical organization of such management was developed based on field work concerning farmers living in the Senegal River delta. Milking is a crucial step of milk management. It helps to shape the "milk sphere", which contains both livestock and people who drive them. The proposed model distinguished different decision making levels: that of milkers/farmers who decided on milked quantities (production), and that of women collectors who decided individually if milked milk was for self-consumption, trade or gift. Besides, the present model helped avoid the common trap of confusing herd manager or concession head with dairy farm head.

Keywords: Milk - Milk products - Milking - Organization Family - Model - Senegal.

\section{Resumen}

Corniaux C. Organización social y zootécnica de la gestión de productos lecheros en medio sahelino: la esfera lechera. Caso del delta del río Senegal

En el Sahel, la concesión es una organización social común pero compleja. La ilustración es dada por la gestión de los productos lecheros en medio pastoril y agro pastoril. Sobre la base de un trabajo de campo llevado a cabo con criadores del delta del río Senegal, se estableció un esquema de la organización social y zootécnica de esta gestión. El ordeño es el momento crucial para la gestión de la leche. Este permite la determinación de los contornos de la esfera lechera, que reagrupa a la vez los animales en producción y los individuos que los gestionan. El modelo propuesto permite distinguir diferentes niveles de decisión: el del pastor-ordeñador que decide las cantidades a ordeñar (producción) y el de los colectores que deciden individualmente el futuro de la leche ordeñada (auto consumo, don, comercio). Por otro lado, nuestro modelo permite evitar la trampa frecuente de asimilar al gestor de hato o el jefe de concesión a un piloto de explotación lechera.

Palabras clave: Leche - Producto lácteo - Ordeño Organización - Familia - Modelo - Senegal. 